\section{The survival of Class $\checkmark$ restorations in general dental practice: part 3, five-year survival}

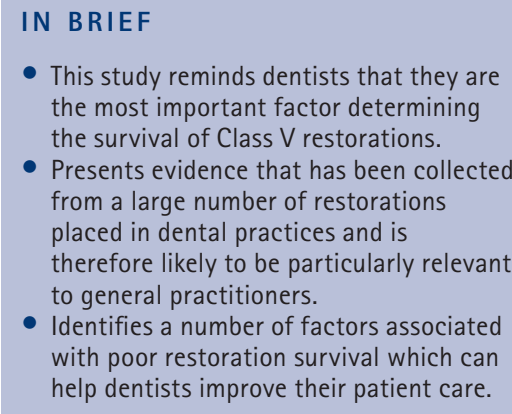

\author{
D. Stewardson, ${ }_{1}^{1}$ S. Creanor, ${ }_{1}^{2}$ P. Thornley, ${ }_{1}^{3}$ T. Bigg, ${ }_{1}^{4}$ C. Bromage, ${ }_{1}^{5}$ \\ A. Browne, ${ }_{1}^{6}$ D. Cottam, ${ }^{7}$ D. Dalby, ${ }^{8}$ J. Gilmour, ${ }^{9}$ J. Horton, ${ }_{1}^{10}$ E. Roberts, ${ }_{1}^{11}$ \\ L. Westoby ${ }^{12}$ and T. Burke ${ }^{13}$
}

- This study reminds dentists that they are the most important factor determining the survival of Class $V$ restorations. Presents evidence that has been collected from a large number of restorations placed in dental practices and is chere likely to be particularly relevant with poor restoration survival which can help dentists improve their patient care.

Objective To evaluate the survival over five years of Class $V$ restorations placed by UK general practitioners, and to identify factors associated with increased longevity. Design Prospective longitudinal cohort multi-centre study. Setting UK general dental practices. Materials and method Ten general dental practitioners each placed 100 Class $V$ restorations of varying sizes, using a range of materials and recorded selected clinical information at placement and recall visits. After five years the data were analysed using the Kaplan-Meier method, log-rank tests and Cox regressions models to identify significant associations between the time to restoration failure and different clinical factors. Results After five years 275/989 restorations had failed (27.8\%), with 116 (11.7\%) lost to follow-up. Cox regression analysis identified that, in combination, the practitioner, patient age, cavity size, moisture contamination and cavity preparation were found to influence the survival of the restorations. Conclusions At least $60.5 \%$ of the restorations survived for five years. The time to failure of Class $V$ restorations placed by this group of dentists was reduced in association with the individual practitioner, smaller cavities, glass ionomer restorations, cavities which had not been prepared with a bur, moisture contamination, increasing patient age, cavities confined to dentine and non-carious cavities.

\section{INTRODUCTION}

Cavities affecting the cervical regions of teeth are a common clinical finding ${ }^{1}$ and may require restoration if associated with

\footnotetext{
${ }^{1 *}$ Lecturer in Conservative Dentistry, University of Birmingham School of Dentistry, St Chads Queensway, Birmingham, B4 6NN; ${ }^{2}$ Lecturer in Health Statistics, Centre for Health and Environmental Statistics, University of Plymouth, Tamar Science Park, Plymouth, Devon, PL6 8BX; ${ }^{3}$ General Dental Practitioner, Walmley Dental Practice, 1 Walmley Close, Sutton Coldfield, West Midlands, B76 1NO; ${ }^{4}$ General Dental Practitioner, Milton Dental Practice, Breakspeare House, Shipton Road, Milton-Under-Wychwood, Oxford, 0X7 6JW; ${ }^{5}$ General Dental Practitioner, Worcester Street Dental Centre, ${ }^{6} 8$ Worcester Street, Stourbridge, West Midlands, DY8 1AY; 6General Dental Practitioner, Evesham Place Dental Practice, 14 Evesham Place, Stratfordupon-Avon, CV37 6HT; ${ }^{7}$ General Dental Practitioner, Cottam \& Cottam Dental Surgery, 1 St Peters Road, Harbourne, Birmingham, B17 OAT; ${ }^{8}$ General Dental Practitioner, Hagley Dental Practice, 157 Worcester Road, Hagley, Stourbridge, West Midlands, DY9 ONW: ${ }^{9}$ General Dental Practitioner, ADP Chasetown, 27 High Street, Chasetown, Burntwood, Staffordshire, WS7 3XE; ${ }^{10}$ General Dental Practitioner, Chelmsley Wood Primary Care Centre, 14 Crabtree Drive, Chelmsley Wood Birmingham, B37 5BU; ${ }^{11}$ General Dental Practitioner, Matthew Gibbons \& Associates Dental Surgery, Neachells Lane, Wolverhampton, WV11 3RF; ${ }^{12}$ General Dental Practitioner, Brighton Villa Dental Care, 7 Bath Street, Hereford, Herefordshire, HR1 2HG; ${ }^{13}$ Professor of Primary Dental Care, University of Birmingham, School of Dentistry, St Chads Queensway, Birmingham, B4 6NN *Correspondence to: Dr Dominic Stewardson Email: d.a.stewardson@bham.ac.uk; Tel: 01212372812
}

Online article number E14

Refereed Paper - accepted 23 February 2012

DOI: $10.1038 /$ sj.bdj.2012.367

${ }^{\circledR}$ British Dental Journal 2012; 212: E14 caries, needed to alleviate sensitivity, improve appearance or prevent enlargement of the lesion. Failure of such Class V restorations appears to be a common occurrence in practice, ${ }^{2}$ which has economic implications both for the dentist and the patient and may undermine the confidence of the patient in the dentist's abilities. A variety of materials and adhesive agents are now suggested for the restoration of Class $\mathrm{V}$ (avities ${ }^{3,4}$ and selecting which will perform best in each situation may present a challenge to the clinician. In the case of non-carious cervical lesions (NCCLs) the aetiology is uncertain and probably multifactorial, ,,6 and without a clear understanding of how a lesion has arisen, it is difficult to specify the desirable physical and mechanical properties required of the restorative material. Laboratory studies can produce information which may suggest how a material will perform clinically, but the correlation between laboratory tests and clinical performance is limited. ${ }^{7,8}$ Controlled clinical studies may provide more relevant information, but many report the results after short periods of observation. ${ }^{9-12}$ The use of such one- and two-year studies may be driven by the 18-month and 2-year periods for provisional and final acceptance of the clinical use of adhesive bonding agents previously used by the American Dental Association. ${ }^{13}$ While a $90 \%$ survival of restorations over two years may be an appropriate screening test before a material is used clinically, restorations should survive for much longer to be acceptable to patients and clinicians ${ }^{14}$ and therefore data from studies of greater duration are needed.

Clinical studies may also be conducted by just one or two experienced clinicians under ideal conditions. Such studies may reveal as much about the ability of these individual operators as they do about the potential performance of the selected materials. ${ }^{15-17}$ Marked differences in the survival of similar restorations have been reported in studies where restorations have been placed by different dentists under the 'real-life' conditions of general practice. ${ }^{18,19}$ Studies of relevant duration conducted in general practice are required to provide information which can supplement and complement the evidence gleaned from these other sources. A more complete and balanced understanding of restoration performance and the factors which influence it may then be gained. In this study therefore, we report on the results of our study of the survival at five years of Class $\mathrm{V}$ restorations placed in general practices and the associations of 
different factors which may be linked with the time to failure.

\section{MATERIALS AND METHOD}

As described in detail in our earlier publication, ${ }^{20}$ ten UK dentists were recruited and each placed 100 Class V restorations in their general practices and recorded selected clinical information on the patient, the cavity and the restoration. Over a fiveyear period the restorations were reviewed when the patients attended for recall visits and the condition, presence or absence of each one was recorded. The data for each of the restorations were entered into an Excel worksheet (Microsoft Corp. Redmond, WA. USA) and then statistical analysis of the data carried out using the computer packages StatXact 8 (Cytel Studio Inc) and SPSS (V16. SPSS Inc. USA). The significance level was set at $\alpha=0.05$.

Using the Kaplan-Meier method, univariate 'associations' were assessed between the time to restoration failure and the following factors:

- The practitioner

- The patient's age, gender and oral hygiene (good, fair, poor). Determination of the oral hygiene and moisture contamination categories were agreed through group discussion. The oral hygiene categories were based on the Plaque Index of Silness and Loe $^{21}$

- Method of payment (privately funded, placed within the National Health Service (NHS), or within an independent funding scheme)

- The tooth: upper or lower, features of the occlusion

- The cavity: condition (carious or noncarious); size (the product of the height and depth of the cavity in $\mathrm{mm}$ ); position - in dentine only or within enamel and dentine, and relative to the gingival margin; cavity preparation (none, use of a bur in a dental handpiece, use of a hand excavator or prophylaxis only with a polishing brush); moisture contamination (subjectively assessed as major, minor or none)

- The restoration: type of material (amalgam, glass ionomer, resin modified glass ionomer, compomer, composite, flowable composite) and, in relation to composite restorations, the type of bonding agent used. The effect of the type of bonding agent on survival of

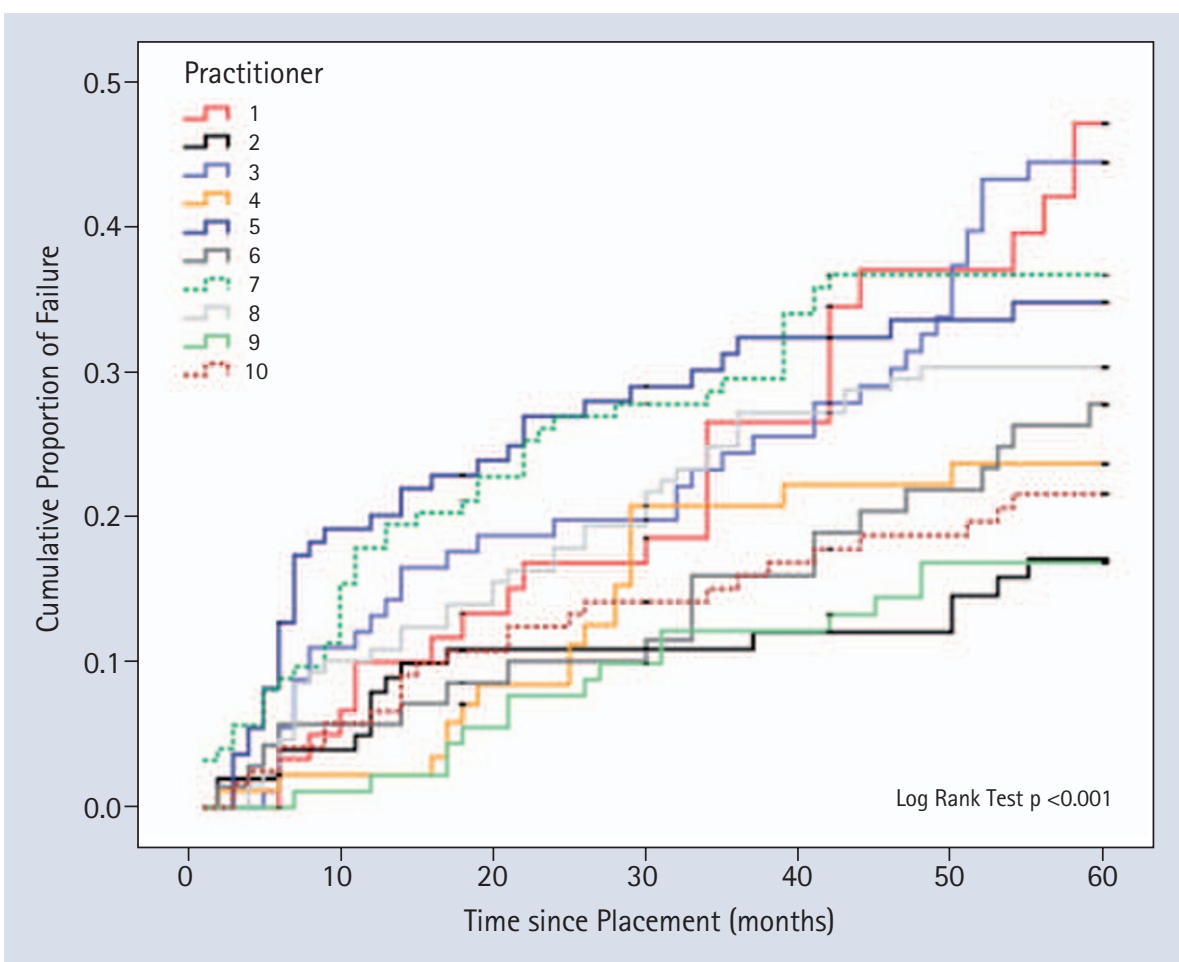

Fig. 1 Kaplan-Meier plot of time to restoration failure for each practitioner. Censoring point markers removed for clarity

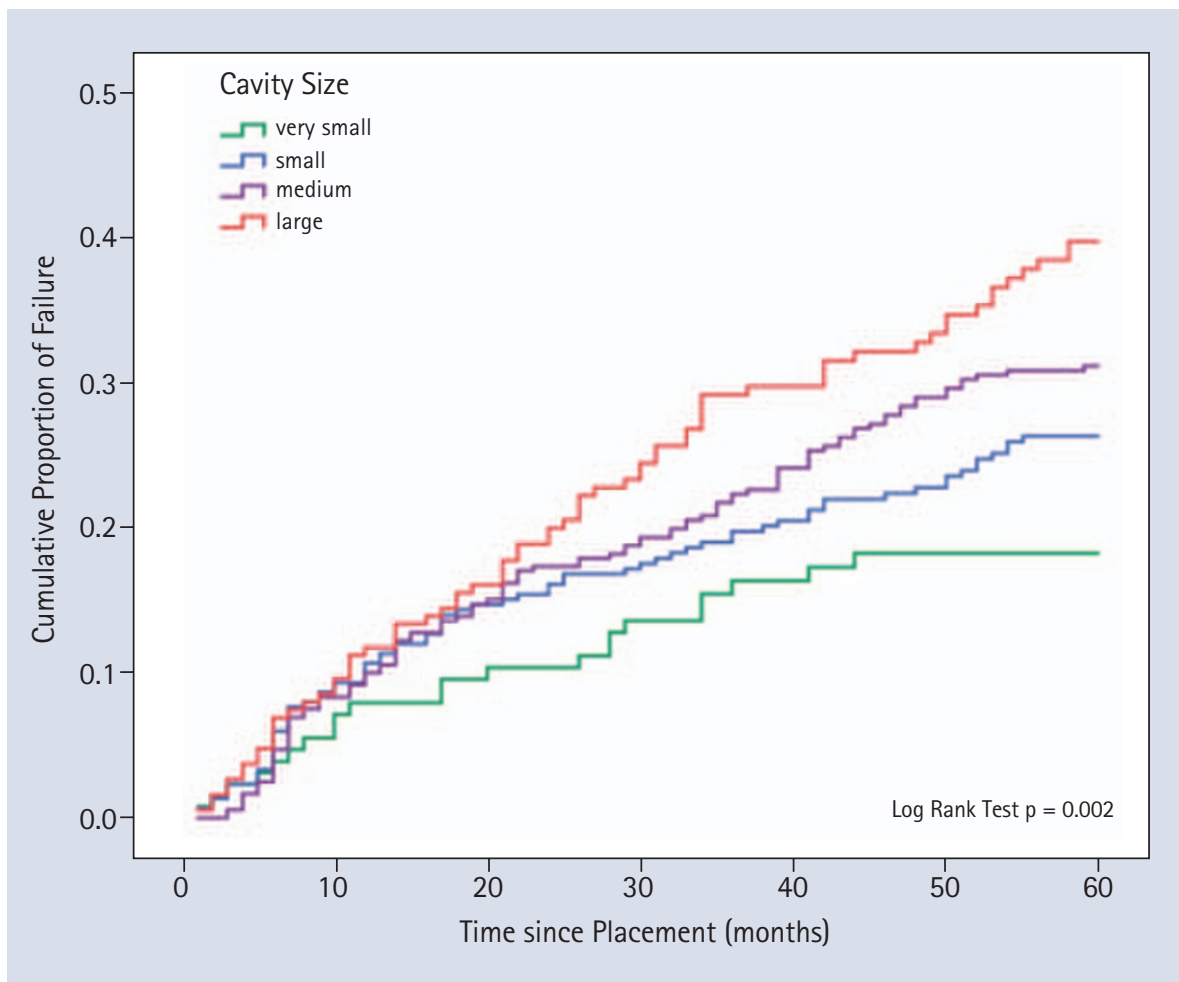

Fig. 2 Kaplan-Meier plot of time to restoration failure for different cavity sizes

composite restorations however will be considered in a later paper.

The log-rank test was used to compare the time to restoration failure between the levels of each of the above factors. In order to have reasonable sample sizes in all levels of each factor, some recoding of factors was required. For completeness, patient age (at time of restoration placement) was categorised ( $\leq 39,40-49,50-59,60-69, \geq 70$ years of age). For factors with more than two levels, follow-up multiple comparisons were made to try to identify which factor levels were significant with regard to differences in time to failure. These 'pair-wise' comparisons 


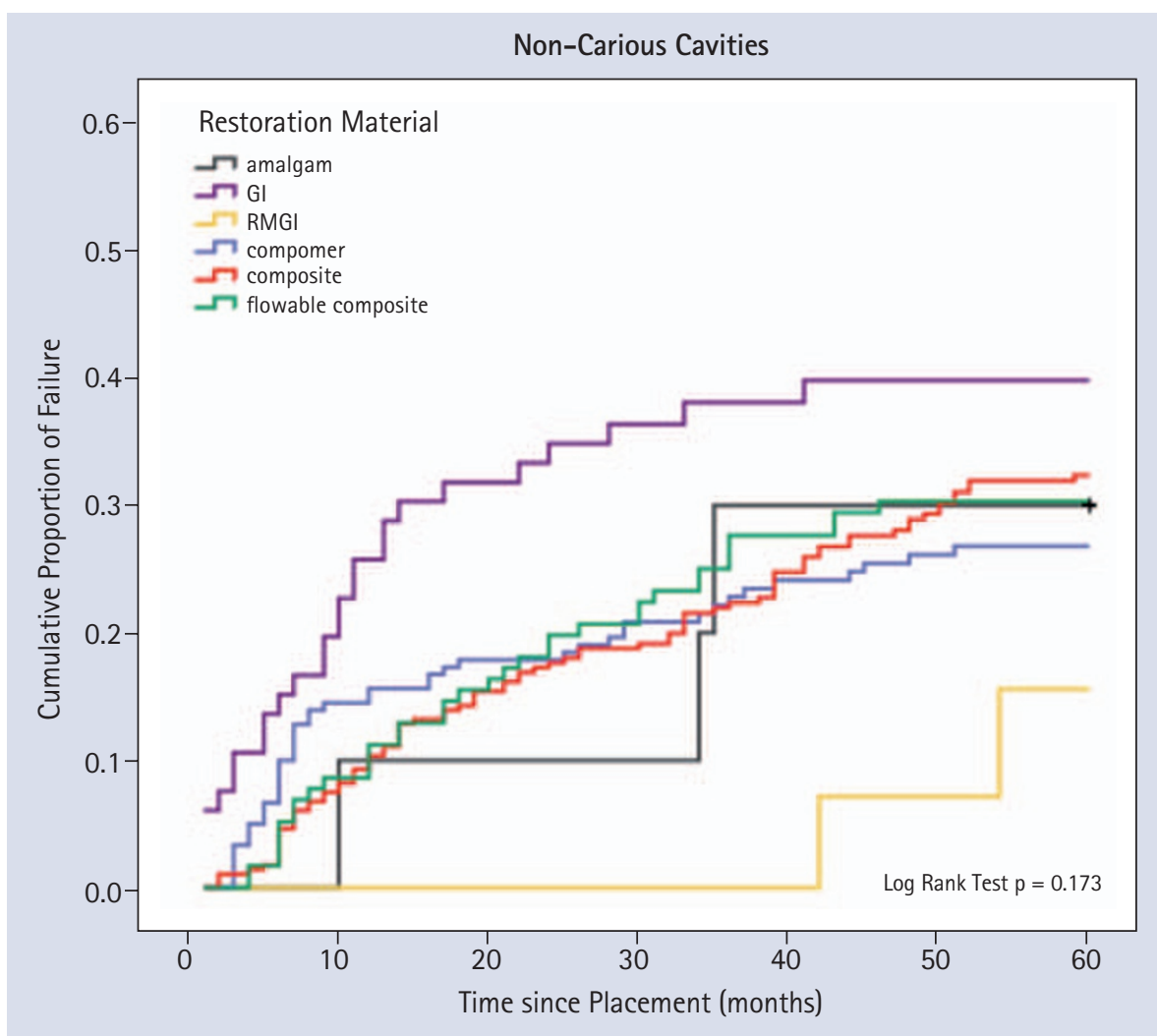

Fig. 3a Kaplan-Meier plot of time to restoration failure for different restoration materials in non-carious cavities

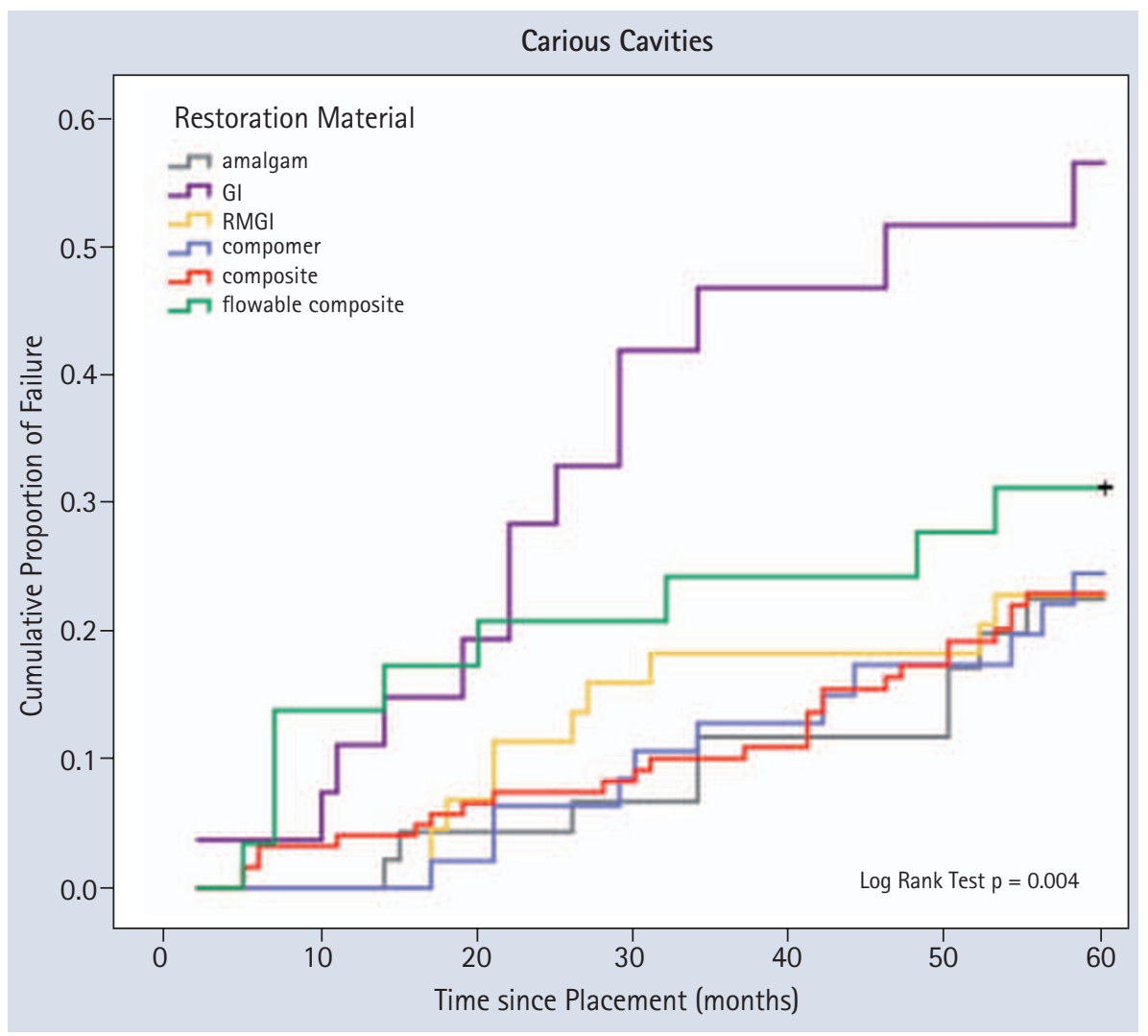

Fig. 3b Kaplan-Meier plot of time to restoration failure for different restoration materials in carious Class V cavities

were not corrected for multiple comparisons given the exploratory nature of this analysis. Further comparison of the restoration failure rates between different factors was undertaken to investigate any significant associations using Pearson's chi-squared tests. Where a significant result was found, follow-up multiple comparisons were made with p-values adjusted using the Bonferroni method to identify where the significant differences had occurred.

Cox regression models were then used to try to identify which combination of the above factors had a significant effect on the time to restoration failure. Patient age was treated as a continuous variable within the Cox regression modelling (all other variables were treated as categorical, with contrasts made to a reference level for each factor). A flexible modelling approach was adopted, with models determined using both forward and backward stepwise methods in an attempt to identify the best subset of factors. Several possible models were examined and the proportional hazards assumption was investigated for independent variables within each possible model by omitting each factor one by one from the model and examining the plot of the log of the cumulative hazard against the log of the time to restoration failure or by examination of the partial residuals (Schoenfeld residuals) where appropriate. Where the proportional hazards assumption was found to be in doubt, alternative models were examined.

\section{RESULTS}

A total of 1,000 restorations were placed. However, the baseline data was incomplete for 11 restorations leaving 989 which were included in the study. By the end of the five-year follow-up, 275 (27.8\%) restorations had failed and 116 (11.7\%) had been lost to follow-up.

The modes of failure were:

- 228 (83\%) complete loss of the restoration

- 15 (5\%) teeth were crowned

- 12 (4\%) teeth were removed or fractured

- $11(4 \%)$ restorations were partially lost

- 9 (3\%) became carious.

\section{Univariable analyses}

Log rank tests identified statistically significant differences in time to restoration failure related to: practitioner $(\mathrm{p}<0.001)$, cavity size ( $p=0.002)$, restoration material $(p=0.002)$, cavity preparation method $(\mathrm{p}=0.004)$, patient age ( $p=0.009$ ), cavity position - in enamel and dentine or just within dentine ( $p=0.012)$ and cavity condition ( $p=0.036)$. Figures 1-7 show the Kaplan-Meier plots of time to restoration failure for each of these 
factors of interest. From visual inspection of the Kaplan-Meier plots, for some of the factors the rate of failure indicated by the slope of the plot was different in the earlier months than in the later months. This was the case in the plots for cavity condition, cavity position, cavity preparation, restoration material and practitioner where failure of restorations rose more rapidly over the initial 18 months than over the remaining period in non-carious cavities, cavities confined to dentine, cavities not prepared with a bur and those restored with glass ionomer and by some of the dentists. Cavity size, in contrast, showed no difference in the slope of the failure plot for the four size categories until after ten months, at which point the slope of the plot for the large cavities became flatter than for the smaller cavities.

Follow-up multiple comparisons were made for factors with more than two levels (Table 1). This shows where the significant differences between factors originated.

\section{Multi-variable analyses excluding practitioner effect}

The first phase of the multi-variable Cox regression modelling did not include a possible effect of practitioner. The best fitting model included the following variables: patient age, cavity position, cavity size, cavity preparation, moisture contamination and restoration material.

The results from this model are shown in Table 2. Patient age was treated as a continuous variable within the Cox regression modelling (all other variables were treated as categorical, with contrasts made to a reference level for each factor).

Increasing patient age was associated with a shorter time to restoration failure (hazard increases by $1 \%$ per additional year on average), after adjusting for the other significant factors. In terms of the other factors, in summary:

- Cavity position: restorations involving dentine only were associated with a shorter time to failure than those involving enamel and dentine (hazard of failure increases by 39\% on average)

- Cavity size: larger cavity sizes were associated with reduced time to restoration failure than very small cavities. For medium sized cavities, the hazard of restoration failure increased on average by $85 \%$ compared to very small cavities, while large cavities

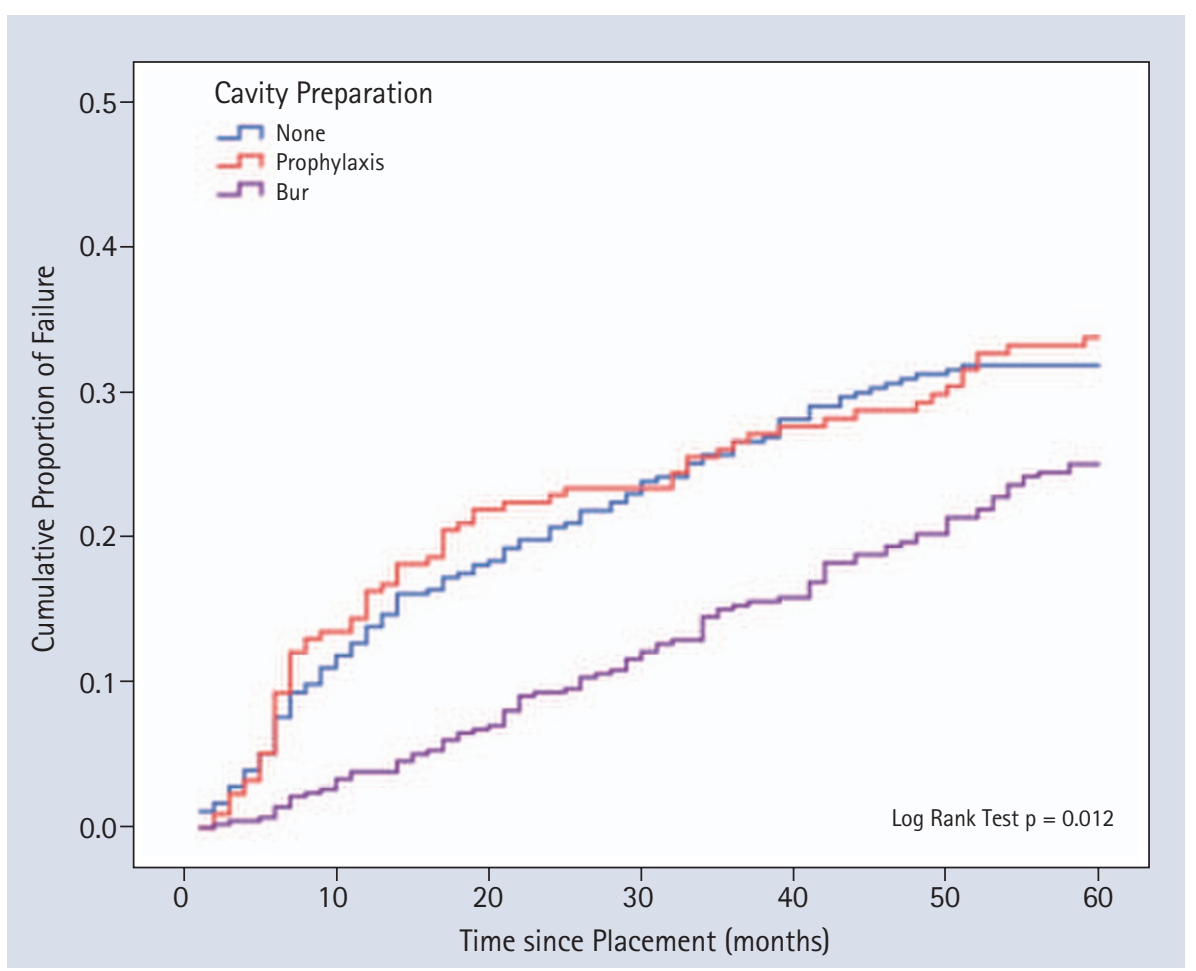

Fig. 4 Kaplan-Meier plot of time to restoration failure for cavity preparation method

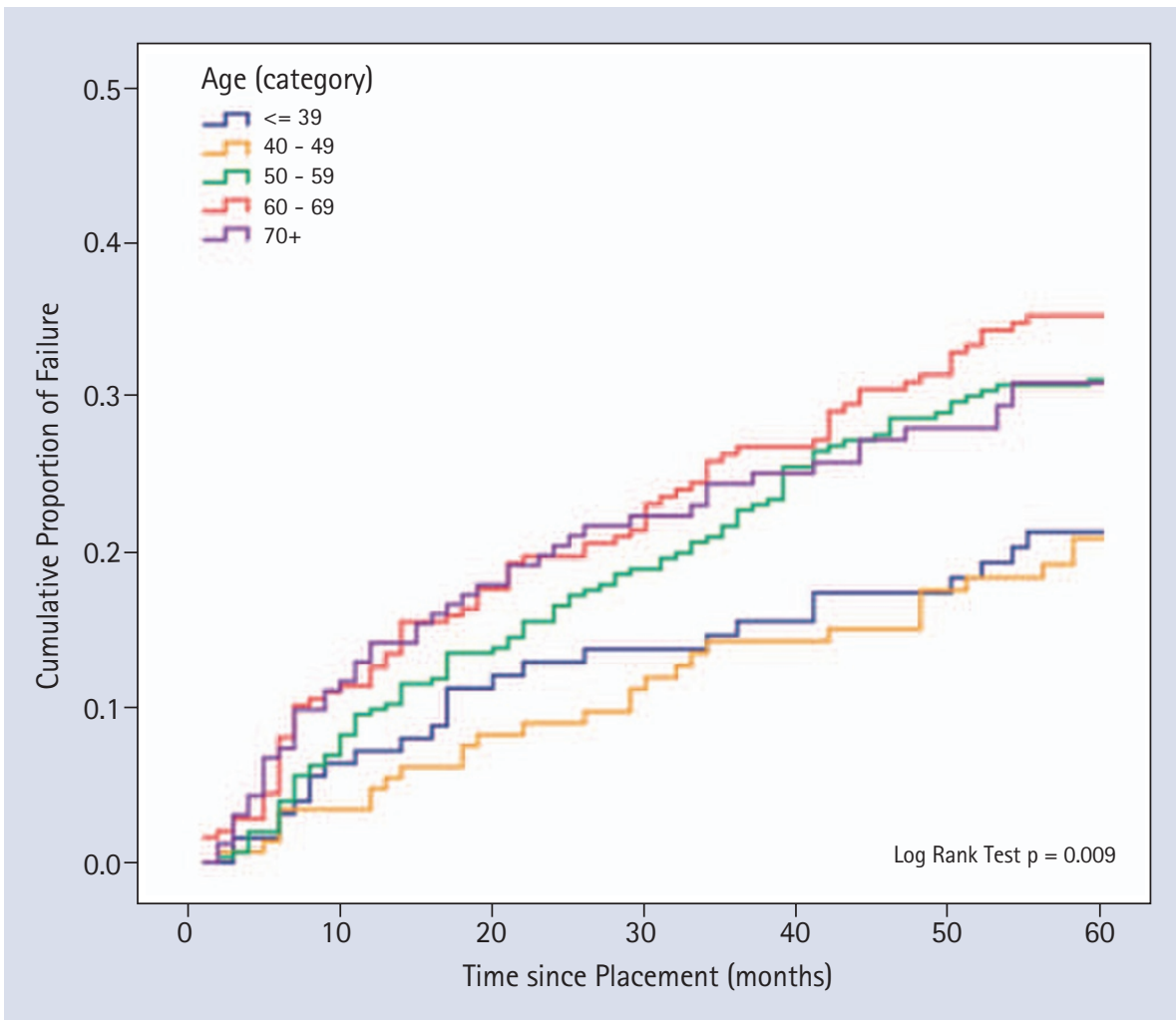

Fig. 5 Kaplan-Meier plot of time to restoration failure for the different categories of patient age

increased the hazard of failure by $165 \%$ on average

- Moisture contamination: minor or major moisture contamination was associated with a shorter time to failure than no moisture (hazard of failure increased by $29 \%$ on average, although it should be noted this was not strictly significant at the $5 \%$ level)

- Preparation method: use of a rotary instrument was associated with an increase in time to restoration failure (hazard of failure reduced by $40 \%$ on average) compared to no preparation 


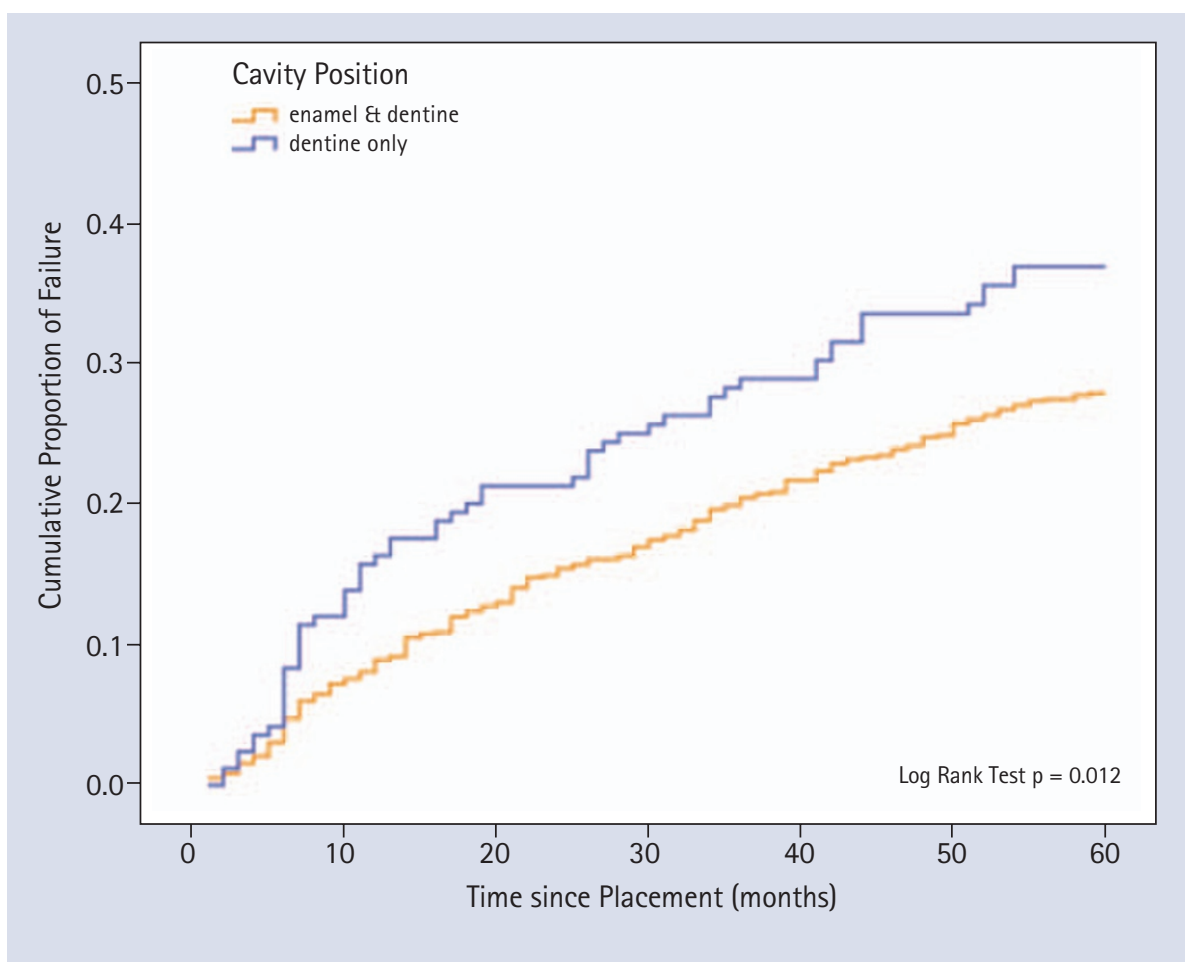

Fig. 6 Kaplan-Meier plot of time to restoration failure for position of cavity

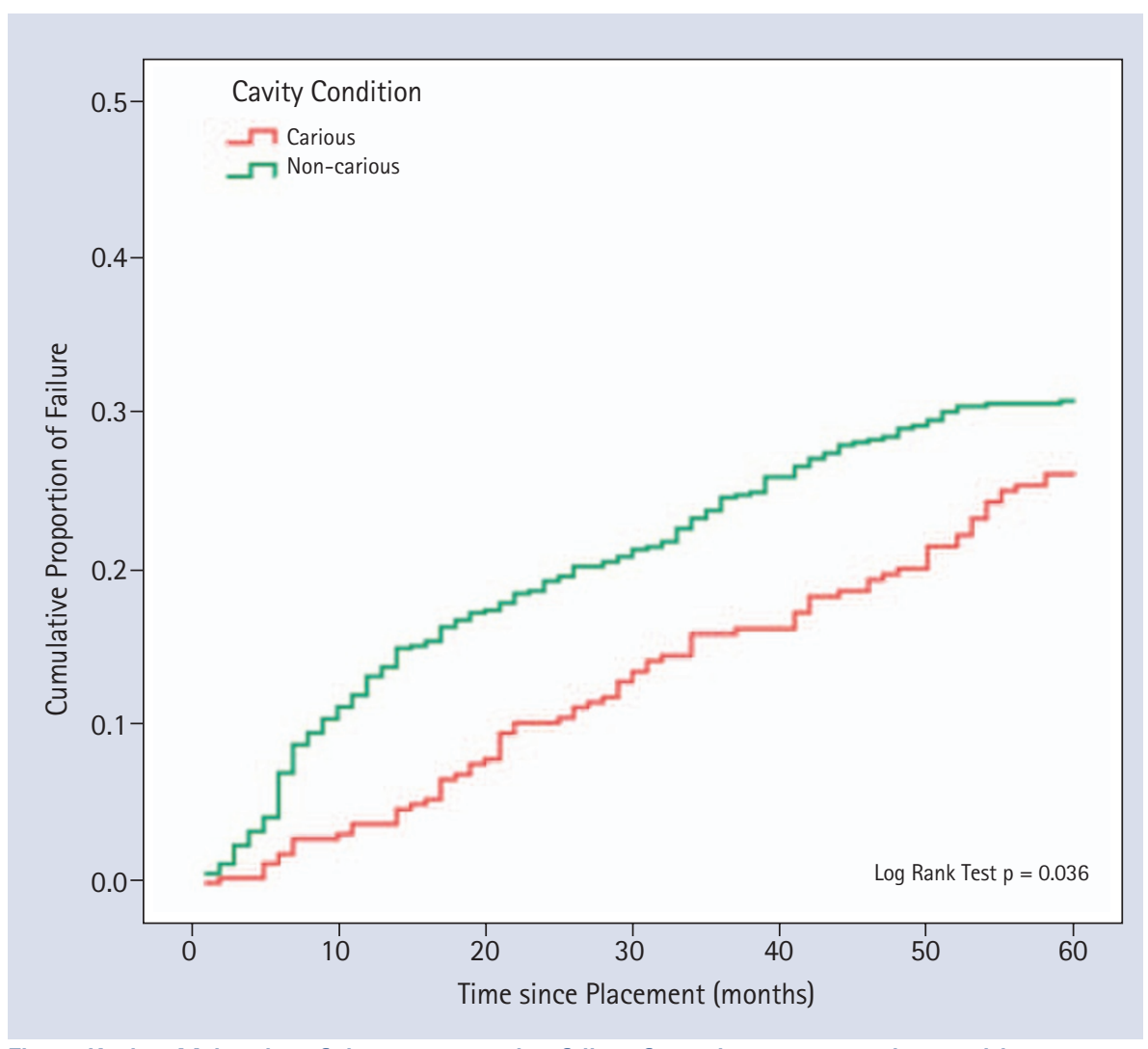

Fig. 7 Kaplan-Meier plot of time to restoration failure for carious or non-carious cavities

Restoration material: the five-year survival of the different materials was: amalgam - 75\%, glass ionomer (GI) - 50.6\%, resin-modified glass ionomer (RMGI) - 78.6\%, compomer - 71.2\%, composite - 68.3\%, flowable composite - 69\%. RMGI was associated with a longer time to restoration failure than GI (hazard reduced by around 60\%) and similarly compomer was associated with a longer time to restoration failure than GI (hazard reduced by around 40\%). Composite was also associated with a longer time to restoration failure than GI (hazard reduced by around 35\%) after adjusting for the other factors in this model.

The proportional hazards assumption for each of the above variables was checked visually and found to be reasonable for all the variables considered.

\section{Multi-variable analyses including practitioner effect}

The second stage of the Cox regression modelling was to consider the effect of practitioner, in addition to all other potential variables. The best fitting model included: patient age, preparation method, moisture contamination, cavity size and practitioner. The results from this model are shown in Table 3.

Increasing patient age was associated with a shorter time to restoration failure (hazard increased by $1.5 \%$ per additional year on average), after adjusting for the other significant factors. In terms of the other factors, in summary:

- Cavity size: as cavity size increased, there was an associated reduction in time to restoration failure. Small cavities increased the hazard of restoration failure on average by $87 \%$ compared to very small cavities, while medium cavities nearly doubled the hazard of restoration failure on average (99\% increase) compared to very small cavities, and large cavities increased the hazard of failure on average by $174 \%$

- Moisture contamination: the presence of minor/major moisture contamination was associated with a shorter time to failure than no moisture contamination (hazard of failure increased by $70 \%$ on average)

- Preparation method: use of rotary instrument/bur was associated with an increase in time to restoration failure (hazard of failure reduced by 54\% on average) compared to no preparation

- Practitioner: practitioners two, six and nine were associated with increased time to restoration failure, in comparison with practitioner one. The five-year survival of restorations ranged from $35 \%$ to $74.5 \%$ for the different practitioners.

The proportional hazards assumption for each of the above variables was checked visually. The assumption was reasonable for patient age and moisture contamination but more doubtful for preparation 
method, cavity size and in particular, practitioner. Given the potential problems with the proportional hazards assumption, variations of the above model were fitted with (a) preparation recorded as having two levels (none/prophylaxis/excavator vs rotary/ bur); (b) cavity size recorded as having two levels (very small/small vs medium/ large); (c) treating cavity size as a continuous variable. Regardless of how each of these variables was considered within the Cox regression modelling, there was little change to the parameter estimates. The proportional hazards assumption was reasonable for preparation method with two levels but not for cavity size with two levels. If cavity size was considered as a continuous variable then on average a one unit increase in cavity size was associated with an increased hazard of failure of $11 \%$.

Because both age and cavity size affected time to survival, the possible association of these two factors was assessed and a significant positive correlation was found between the size of the cavity and the age of the patient that is, cavity size increased with increasing patient age (Pearson bi-variate correlation coefficient, two-tailed, 0.108; C.I. 0.046-0.169; $\mathrm{p}<0.01$ ).

\section{Cavity condition and material}

There was evidence of a statistically significant association between material type used and whether the cavity was carious or noncarious (chi-square test $\mathrm{p}<0.001$ ). Follow-up comparisons showed that greater proportions of the carious cavities were filled with amalgam or RMGI than other materials. The data were therefore separated into noncarious and carious cavities and univariate associations assessed between the time to restoration failure and restoration material. For both cavity conditions, the time to failure of GI restorations was shorter than for the other materials, but the log rank tests indicated that this was only significant for carious cavities $(p=0.004)$ and not for noncarious cavities ( $p=0.173$ ).

For carious cavities there was insufficient evidence of a significant difference in the time to failure of restorations associated with being prepared with a bur or not ( $p=0.09$ ), but only 16 carious cavities were not prepared with a bur. With non-carious cavities, the time to failure of restorations placed in cavities which had not been prepared was

Table 1 Follow-up multiple comparisons for significant Log Rank tests (excluding practitioner). Significant differences indicated in bold

\begin{tabular}{|c|c|c|c|c|}
\hline \multirow[t]{2}{*}{ Factor } & \multicolumn{3}{|l|}{ Comparison } & \multirow{2}{*}{$\frac{P \text {-value }}{0.825}$} \\
\hline & $40-49$ years & - & $\leq 39$ years & \\
\hline \multirow{9}{*}{$\begin{array}{l}\text { Age } \\
\text { categorised }\end{array}$} & $50-59$ years & - & $\leq 39$ years & 0.054 \\
\hline & $60-69$ years & - & $\leq 39$ years & 0.008 \\
\hline & $70+$ years & - & $\leq 39$ years & 0.062 \\
\hline & $50-59$ years & - & $40-49$ years & 0.022 \\
\hline & $60-69$ years & - & $40-49$ years & 0.002 \\
\hline & $70+$ years & - & $40-49$ years & 0.027 \\
\hline & $60-69$ years & - & $50-59$ years & 0.275 \\
\hline & $70+$ years & - & $50-59$ years & 0.858 \\
\hline & $70+$ years & - & $60-69$ years & 0.469 \\
\hline \multirow{6}{*}{ Cavity size } & Small & - & Very small & 0.104 \\
\hline & Medium & - & Very small & 0.014 \\
\hline & Large & - & Very small & $<0.001$ \\
\hline & Medium & - & Small & 0.239 \\
\hline & Large & - & Small & 0.006 \\
\hline & Large & - & Medium & 0.065 \\
\hline \multirow{3}{*}{$\begin{array}{l}\text { Preparation } \\
\text { method }\end{array}$} & Prophylaxis/excavator & - & None & 0.674 \\
\hline & Rotary/bur & - & None & 0.005 \\
\hline & Rotary/bur & - & Prophylaxis/excavator & 0.003 \\
\hline \multirow{15}{*}{$\begin{array}{l}\text { Restoration } \\
\text { material }\end{array}$} & $\mathrm{Gl}$ & - & Amalgam & 0.004 \\
\hline & RMGI & - & Amalgam & 0.776 \\
\hline & Compomer & - & Amalgam & 0.462 \\
\hline & Composite & - & Amalgam & 0.289 \\
\hline & Flowable composite & - & Amalgam & 0.206 \\
\hline & RMGI & - & $\mathrm{Gl}$ & 0.002 \\
\hline & Compomer & - & $\mathrm{Gl}$ & 0.001 \\
\hline & Composite & - & $\mathrm{Gl}$ & 0.001 \\
\hline & Flowable composite & - & $\mathrm{Gl}$ & 0.020 \\
\hline & Compomer & - & RMGI & 0.312 \\
\hline & Composite & - & RMGI & 0.159 \\
\hline & Flowable composite & - & RMGI & 0.124 \\
\hline & Composite & - & Compomer & 0.647 \\
\hline & Flowable composite & - & Compomer & 0.414 \\
\hline & Flowable composite & - & Composite & 0.631 \\
\hline
\end{tabular}

significantly reduced compared with those which had been prepared ( $p=0.008)$.

\section{DISCUSSION}

This series of three papers ${ }^{20,22}$ demonstrates that general practitioners working in partnership with academics in a group such as BRIDGE (Birmingham Research In General Dental practice) have the capacity to initiate, design and carry out substantial research projects. It also illustrates the potential of practice-based research to generate larger amounts of relevant information than is usually produced from other clinical study settings. Unlike in medicine, however, there is little value or financial support given to practice based-research. Along with its merits, there are limitations to this current research. It was not a randomised controlled study and the practitioners were 


\begin{tabular}{|c|c|c|c|}
\hline Factor & & Relative hazard of failure $(95 \% \mathrm{CI})$ & P-value \\
\hline Age & & 1.014 (1.004 to 1.022$)$ & 0.006 \\
\hline \multirow{2}{*}{ Cavity position } & Dentine only* & - & \multirow{2}{*}{0.041} \\
\hline & Enamel + dentine & 1.390 (1.013 to 1.906$)$ & \\
\hline \multirow{4}{*}{ Cavity size } & Very small ${ }^{*}$ & - & \multirow{4}{*}{$<0.001$} \\
\hline & Small & 1.487 (0.920 to 2.402$)$ & \\
\hline & Medium & 1.845 (1.159 to 2.935$)$ & \\
\hline & Large & 2.648 (1.619 to 4.331$)$ & \\
\hline \multirow{2}{*}{$\begin{array}{l}\text { Moisture } \\
\text { contamination }\end{array}$} & None $^{*}$ & - & \multirow{2}{*}{0.068} \\
\hline & Minor/major & 1.285 (0.982 to 1.682$)$ & \\
\hline \multirow{3}{*}{$\begin{array}{l}\text { Preparation } \\
\text { method }\end{array}$} & None $^{*}$ & - & \multirow{3}{*}{0.001} \\
\hline & Prophylaxis/excavator & 1.003 (0.731 to 1.376$)$ & \\
\hline & Rotary/bur & 0.596 (0.440 to 0.808$)$ & \\
\hline \multirow{6}{*}{$\begin{array}{l}\text { Restoration } \\
\text { material }\end{array}$} & $\mathrm{Gl}^{*}$ & - & \multirow{6}{*}{0.041} \\
\hline & Amalgam & 0.567 (0.288 to 1.115$)$ & \\
\hline & RMGI & 0.396 (0.205 to 0.766$)$ & \\
\hline & Compomer & 0.599 (0.394 to 0.911) & \\
\hline & Composite & 0.644 (0.437 to 0.950$)$ & \\
\hline & Flowable composite & 0.804 (0.503 to 1.285$)$ & \\
\hline
\end{tabular}

not obliged to place specific materials, but could instead select restoratives according to their own clinical preferences. In addition, some patients received more than one Class V restoration. This imposes some limits on the conclusions which may be drawn. Nonetheless, the study design does reflect what occurs in daily practice, where general dentists are subject to time constraints and restore teeth in conditions which may be less than ideal. It is perhaps not surprising therefore, that one of the most important factors influencing survival time was the practitioner who placed the restoration and this should be borne in mind when considering evidence on the performance of restorations provided by only one or two clinicians (who may indeed be specialists). ${ }^{23,24}$

Since the choice of material was theirs to make, it would be expected that each dentist would use materials with which they were familiar and felt confident to handle. It is evident (from our initial paper ${ }^{20}$ ) that the choice of material differed among the ten practitioners and was influenced by the condition of the cavity. However, confidence does not equate to competence and the difference in the proportion of failures between clinicians suggests that differences in individual practical skill and knowledge play a major role in the length of service of Class V restorations. Practitioners should be encouraged to conduct similar audits of their own performance and compare with others to identify if their standards are adequate. If indicated, they should then seek appropriate training and advice to improve their quality of care.

When compared with other recent studies of similar duration, this study has compared a wider range of materials, included a greater number of restorations of each type ${ }^{23-28}$ and had fewer restorations lost to follow-up than in three of these aforementioned studies. ${ }^{23,26,27}$ However, only $68 \%$ of composite restorations survived to five years in this study compared to proportions of $100 \%,{ }^{24,28} 96 \%,{ }^{27} 92 \%{ }^{23}$ and $52 \% 0^{27}$ reported by others. Studies comparing RMGI with composite $^{27}$ or compomer ${ }^{25}$ report greater five-year survival for RMGI. The results of our study show the same trends although the relative proportions differ.

Within this study (even after allowing for differences between practitioners), restoration failure rate increased with age of the patients. The effect of age on restoration longevity has been identified in other studies ${ }^{29,30}$ involving restorations in different locations on teeth, but the reason for this has not been established. NCCLs may have been present for longer in older patients increasing the degree of dentine sclerosis present and reducing the ability to bond to these lesions. ${ }^{31}$ This may explain the decreased survival of such Class V restorations but not the effect of age on all restorations. It may simply be related to the size of the lesions, since it was established that increasing cavity size was associated with a shorter time to failure and also that there was a positive correlation between increasing patient age and increasing cavity size. Age-related changes in the structure of teeth may also be a factor. Over time, dentine tubules decrease in diameter and number ${ }^{32}$ with an associated decrease in permeability, ${ }^{33}$ water content ${ }^{34}$ and altered mechanical properties of dentine. ${ }^{35}$ The relative contribution of all these factors would be difficult to determine, but the practical significance is that clinicians need to modify their approach when restoring the teeth of older patients to provide longer lasting restorations.

In the first Cox regression model, restorations in cavities extending across both enamel and dentine survived longer than those confined to dentine. Enamel is a more reliable substrate to which to bond and where an adhesive material can be extended across an area of enamel, better retention would be the expected result. This effect, which reached a significant level in the two-year data, becomes more evident after five years where the survival plots show a reduced time to survival of restorations placed into dentine cavities.

There is much debate and interest among clinicians as to which is the 'best' restorative material for different situations. Indeed, one of the principle aims of this study was to try to identify which material should be recommended for restoring Class V cavities. RMGI restorations performed well over five years in comparison with the other tooth-coloured materials, which concurs with the findings of other studies of similar duration. . $5,27^{2}$ Although the different materials used did show some differences in survival, with a greater proportion of GI restorations failing over the five-year period and a greater proportion of RMGI and amalgam restorations surviving, this difference was only 
significant for carious cavities. For cavities where caries was not the cause, the practitioner had a greater influence on survival than the choice of material. The preparation of the cavity also exerted a significant effect on survival. Preparation using a rotary bur significantly increased the time to failure of NCCLs. With hindsight, it would have been desirable to record what extent of preparation with a bur had been carried out, as this could have ranged from a simple roughening of the surface to the creation of a macroretentive cavity. As these lesions required no removal of diseased tissue, it is more likely that only surface roughening had been performed. Despite the success reported in some studies where a 'no preparation' approach had been followed, ${ }^{15}$ a recent meta-analysis $^{36}$ also identified the positive effect on Class V restoration survival associated with roughening of the cavity base.

Although the strength of evidence on restoration material presented here is limited, it does suggest that the apparent ease of use of conventional glass ionomer may not predictably translate into the production of a durable restoration and that a more careful placement technique, including appropriate cavity preparation, is needed..$^{37}$ Similarly, consideration should perhaps be given to using RMGI more frequently in the restoration of NCCLs, as other studies have also shown high retention rates in comparison to other materials. ${ }^{38,39}$

Occlusal factors are considered by many to be important in the aetiology of NCCLs ${ }^{40-}$ ${ }^{42}$ and in the success of restorations..$^{30}$ The use of low-modulus materials is also recommended by some to prevent detachment of adhesive restorations resulting from tooth flexure. ${ }^{43,44}$ This study, however, did not identify any statistically significant effect of any of the occlusal factors recorded on time to failure, which supports the findings of those who discount the influence of occlusal loading as anything more than a co-initiating factor. ${ }^{37,56}$ No evidence of an association between the elastic modulus of a restorative material and time to failure was found, as there was no difference observed between the Kaplan-Meier plots of either of the two composite types and compomer, while glass ionomer, the material with the lowest modulus of elasticity, recorded the greatest proportion of failures. The issue of elastic modulus is somewhat complicated as materials within one classification may have

Table 3 Results of Cox Regression Model, including practitioner, with p-value contrasts made to a reference level for each factor (asterisked)

\begin{tabular}{|c|c|c|c|}
\hline Factor & & $\begin{array}{l}\text { Relative hazard of failure } \\
(95 \% \mathrm{Cl})\end{array}$ & P-value ${ }^{*}$ \\
\hline Age & & 1.015 (1.006 to 1.024$)$ & 0.001 \\
\hline \multirow{4}{*}{$\begin{array}{l}\text { Cavity } \\
\text { size }\end{array}$} & Very small* & - & \multirow{4}{*}{0.003} \\
\hline & Small & 1.870 (1.115 to 3.138$)$ & \\
\hline & Medium & 1.991 (1.215 to 3.260$)$ & \\
\hline & Large & 2.739 (1.611 to 4.656$)$ & \\
\hline \multirow{2}{*}{$\begin{array}{l}\text { Moisture } \\
\text { contamination }\end{array}$} & None $^{*}$ & - & \multirow{2}{*}{0.002} \\
\hline & Minor/major & 1.704 (1.224 to 2.371$)$ & \\
\hline \multirow{3}{*}{$\begin{array}{l}\text { Preparation } \\
\text { method }\end{array}$} & None $^{*}$ & - & \multirow{3}{*}{$<0.001$} \\
\hline & Prophylaxis/excavator & 0.916 (0.618 to 1.357 ) & \\
\hline & Rotary/bur & 0.457 (0.335 to 0.623$)$ & \\
\hline \multirow{10}{*}{ Practitioner } & $1^{*}$ & - & \multirow{10}{*}{$<0.001$} \\
\hline & 2 & 0.340 (0.170 to 0.678$)$ & \\
\hline & 3 & 1.485 (0.786 to 2.805 ) & \\
\hline & 4 & 0.678 (0.353 to 1.304 ) & \\
\hline & 5 & 0.920 (0.514 to 1.650$)$ & \\
\hline & 6 & 0.521 (0.276 to 0.981$)$ & \\
\hline & 7 & 1.476 (0.830 to 2.625$)$ & \\
\hline & 8 & 0.941 (0.516 to 1.716$)$ & \\
\hline & 9 & 0.480 (0.240 to 0.961$)$ & \\
\hline & 10 & 0.529 (0.273 to 1.025$)$ & \\
\hline
\end{tabular}

quite distinct mechanical properties and so it cannot necessarily be assumed, for example, that all composites have a significantly higher elastic modulus than all RMGIs.

When compared with the findings of our previous analysis conducted after two years, the results have suggested that most of the factors influencing the time to failure across five years were the same as the factors identified as influencing the probability of failure within two years of placement, ${ }^{22}$ although the rate of failure was not consistent over the five years. Increasing cavity size did not appear to affect two-year failure but reduced the time to failure of restorations over five years. This may be because larger cavities can have a longer margin or that filling a bigger cavity requires more skill, but as stated previously, cavity size is also linked to patient age and thus possible changes in the dentine.

\section{CONCLUSIONS}

Overall, at least $60.5 \%$ of the Class V restorations placed by this group of dentists survived to five years. The time to failure of Class $\mathrm{V}$ restorations placed by this group of dentists was reduced in association with the individual practitioner, smaller cavities, glass ionomer restorations, moisture contamination, cavities which had not been prepared with a bur, increasing patient age, cavities in dentine only and non-carious cavities. Greater durability of these frequently placed restorations can be achieved principally by improving operator skill, followed by cavity preparation and appropriate material handling.

This work was supported by a grant from the British Dental Association Shirley Glasstone Hughes Memorial Fund.

1. Levitch L C, Bader J D, Shugars D A, Heymann H O. Non-carious cervical lesions. J Dent 1994; 22: 195-207.

2. Perez $C$ R. Alternative technique for $C$ lass $V$ resin composite restorations with minimum finishing/ polishing procedures. Oper Dent 2010; 35: 375-379.

3. Summit J B, Williams Robins, J, Hilton T J, Schwartz R S. Fundamentals of operative dentistry: a contemporary approach. 3rd ed. Chicago: Quintessence Publishing Co Inc., 2006.

4. Pecie R, Krejci I, Garcia-Godoy F, Bortolotto T. Noncarious cervical lesions (NCCL) -a clinical concept based on the literature review. Part 2: 
restoration. Am J Dent 2011; 24: 183-192.

5. Bartlett D W, Shah P. A critical review of noncarious cervical (wear) lesions and the role of abfraction, erosion, and abrasion. J Dent Res 2006: 85: 306-312.

6. Litonjua L A, Andreana S, Bush P J, Tobias TS Cohen R E. Noncarious cervical lesions and abfractions: a re-evaluation. J Am Dent Assoc 2003; 134: 845-850.

7. Van Meerbeek B, Peumans M, Poitevin A et al. Relationship between bond-strength tests and clinical outcomes. Dent Mater 2010; 26: e100-121.

8. Heintze S D. Systematic reviews: I. The correlation between laboratory tests on marginal quality and bond strength. II. The correlation between marginal quality and clinical outcome. J Adhes Dent 2007; 9 (Suppl 1): 77-106.

9. Abdalla A I, El Sayed H Y. Clinical evaluation of a self-etch adhesive in non-carious cervical lesions. Am J Dent 2008; 21: 327-330.

10. Van Landuyt K L, Peumans M, Fieuws S et al. A randomized controlled clinical trial of a HEMA-free all-in-one adhesive in non-carious cervical lesions at 1 year. J Dent 2008; 36: 847-855.

11. Onal B, Pamir T. The two-year clinical performance of esthetic restorative materials in noncarious cervical lesions. J Am Dent Assoc 2005; 136: 1547-1555.

12. Turkun L S, Celik E U. Noncarious Class V lesions restored with a polyacid modified resin composite and a nanocomposite: a two-year clinical trial. J Adhes Dent 2008; 10: 399-405.

13. American Dental Association Council on Dental Materials, Instruments and Equipment. Revised American Dental Association acceptance program guidelines for dentin and enamel adhesive materials. Chicago: American Dental Association, 1994.

14. Steele J. NHS dental services in England: an independent review led by Professor Jimmy Steele. London: Department of Health, 2009.

15. McCoy R B, Anderson M H, Lepe $X$, Johnson G H. Clinical success of Class $V$ composite resin restorations without mechanical retention. J Am Dent Assoc 1998; 129: 593-599.

16. van Dijken J W. Pallesen U. Long-term dentin retention of etch-and-rinse and self-etch adhesives and resin-modified glass ionomer cement in non-carious cervical lesions. Dent Mater 2008; 24: 915-922.

17. Folwaczny M, Mehl A, Kunzelmann K H, Hickel R. Clinical performance of a resin-modified glass-ionomer and a compomer in restoring noncarious cervical lesions. 5-year results. Am J Dent 2001; 14: 153-156.

18. Smales R J, Ng K K. Longevity of a resin-modified glass ionomer cement and a polyacid-modified resin composite restoring non-carious cervical lesions in a general dental practice. Aust Dent J 2004; 49: 196-200.

19. Köhler B, Rasmusson C G, Odman P. A five-year clinical evaluation of Class II composite resin restorations. J Dent 2000; 28: 111-116.

20. Stewardson D, Thornley P, Bigg T et al. The survival of Class $V$ restorations in general dental practice. Part 1, baseline data. Br Dent J 2010; 208: E17; discussion 406-407.

21. Silness J, Loe H. Periodontal disease in pregnancy. II. Correlation between oral hygiene and periodontal condtion. Acta Odontol Scand 1964; 22: 121-135.

22. Stewardson D A, Thornley P, Bigg T et al. The survival of Class $\vee$ restorations in general dental practice. Part 2, early failure. Br Dent J 2011; 210: E19.

23. Burrow M F, Tyas M J. Five-year clinical evaluation of One-Up Bond $\mathrm{F}$ in non-carious cervical lesions. Am J Dent 2007; 20: 361-364.

24. Kubo S, Kawasaki K, Yokota H, Hayashi Y. Five-year clinical evaluation of two adhesive systems in noncarious cervical lesions. J Dent 2006; 34: 97-105.

25. Loguercio A D, Reis A, Barbosa A N, Roulet J F. Fiveyear double-blind randomized clinical evaluation of a resin-modified glass ionomer and a polyacidmodified resin in noncarious cervical lesions. J Adhes Dent 2003; 5: 323-332.

26. Demirci M, Sancakli H S, Uysal O. Clinical evaluation of a polyacid-modified resin composite (Dyract) in Class V carious lesions: 5-year results. Clin Oral Investig 2008; 12: 157-163.

27. Franco E B, Benetti A R, Ishikiriama S K et al. 5-year clinical performance of resin composite versus resin modified glass ionomer restorative system in non-carious cervical lesions. Oper Dent 2006: 31: 403-408.

28. Peumans M, De Munck J, Van Landuyt K, Lambrechts P, Van Meerbeek B. Five-year clinical effectiveness of a two-step self-etching adhesive. J Adhes Dent 2007; 9: 7-10.

29. Burke F J, Lucarotti PS, Holder R L. Outcome of direct restorations placed within the general dental services in England and Wales (part 2): variation by patients' characteristics. J Dent 2005; 33: 817-826.
30. Heymann H O, Sturdevant J R, Bayne S, Wilder A D, Sluder T B, Brunson W D. Examining tooth flexure effects on cervical restorations: a two-year clinical study. J Am Dent Assoc 1991; 122: 41-47.

31. Tay F R, Pashley D H. Resin bonding to cervical sclerotic dentin: a review. J Dent 2004; 32: 173-196.

32. Carrigan P J, Morse D R, Furst M L, Sinai I H. A scanning electron microscopic evaluation of human dentinal tubules according to age and location. J Endod 1984; 10: 359-363.

33. Tagami J, Hosoda H, Burrow M F, Nakajima M. Effect of aging and caries on dentin permeability. Proc Finn Dent Soc 1992; 88 (Suppl 1): 149-154.

34. Toto P D, Kastelic E F, Duyvejonck K J, Rapp G W. Effect of age on water content in human teeth. J Dent Res 1971; 50: 1284-1285.

35. Arola D, Reprogel R K. Effects of aging on the mechanical behaviour of human dentin. Biomaterials 2005; 26: 4051-4061.

36. Heintze S D, Ruffieux C, Rousson V. Clinical performance of cervical restorations - a meta-analysis. Dent Mater 2010; 26: 993-1000.

37. Michael J A, Townsend G C, Greenwood L F, Kaidonis $J$ A. Abfraction: separating fact from fiction. Aust Dent J 2009; 54: 2-8.

38. Peumans M, Kanumilli P, De Munck J, Van Landuyt K, Lambrechts P, Van Meerbeek B. Clinical effectiveness of contemporary adhesives: a systematic review of current clinical trials. Dent Mater 2005; 21: 864-881.

39. Sidhu S K. Clinical evaluations of resin-modified glass-ionomer restorations. Dent Mater 2010; 26: 7-12.

40. Lee W C, Eakle W S. Stress-induced cervical lesions: review of advances in the past 10 years. J Prosthet Dent 1996; 75: 487-494

41. Miller N, Penaud J, Ambrosini P, Bisson-Boutelliez C Briancon S. Analysis of etiologic factors and periodontal conditions involved with 309 abfractions. J Clin Periodontol 2003; 30: 828-832.

42. Takehara J, Takano T, Akhter R, Morita M. Correlations of noncarious cervical lesions and occlusal factors determined by using pressuredetecting sheet. J Dent 2008; 36: 774-779.

43. Blunck U. Improving cervical restorations: a review of materials and techniques. J Adhes Dent 2001; 3: 33-44.

44. Wood I, Jawad Z, Paisley C, Brunton P. Non-carious cervical tooth surface loss: a literature review. J Dent 2008; 36: 759-766. 